\title{
Scene setter: the importance of taking a systems approach to person centred care
}

\author{
Stephen Leyshon deputy director, healthcare, strategic research and innovation, DNV GL, Stephen \\ McAdam global director, healthcare, business assurance, DNV GL
}

\begin{abstract}
Since the end of the second world war healthcare has changed greatly. Advances in treatment mean that the average life expectancy in the UK, for example, has grown by 20 years. But this has come at a cost. Health systems are significantly more complex, with care becoming ever more subspecialised. Increasing numbers of health and social care professionals are involved in each patient's journey through the

system-reflecting the changing needs of populations that are ageing and living longer with comorbid chronic conditions. Within this complexity the service user risks getting lost as different parts of the health and social care system struggle to coordinate with each other. Too often services are delivered in ways that fit with abstract policy ambitions rather than with what patients need or want. In short, existing system models are unsustainable. ${ }^{1}$
\end{abstract}

Yet as the examples in this supplement show, service users, health professionals, managers, and policy makers are proving that change is both desired and possible. In person centred care, the system is redesigned to focus on the service user's experience. Patients, practitioners, providers, and policy makers become equal and active partners in coproducing the health journey. Each brings a different type of experience and expertise to the care process, and together they cocreate a culture to drive change. In doing so, improvements in costs, health status, patient knowledge, adherence with treatment plans, as well as patient and practitioner satisfaction are achievable..$^{2-5}$

Given the benefits of person centred care, the question is why it is not standard practice. ${ }^{6}$ To make the leap from ambition to a reality for all requires a move beyond standalone projects and initiatives to the adoption of systems thinking. Systems thinking is an approach to improvement that views challenges to quality as part of a wider, dynamic structure-looking for patterns of distributed risk rather than fragments or individual episodes. It involves more than a reaction to a particular outcome or event; it requires a deeper understanding of the distribution, linkages, and relationships among the processes that characterise the entire system. ${ }^{7}$ Common in other safety critical and complex sectors, systems thinking focuses on:
- Establishing policies that set clear and explicit goals and directions regarding quality (including defining what quality means for an organisation)

- Mapping processes and identifying indicators to monitor those processes (including how they connect within and between organisations)

- Identifying and assessing risks to human, technological, and organisational safety and performance (including how these are distributed within and across organisations and who owns them)

- Creating pre-emptive and mitigation controls to deliver safe and consistently reliable results

- Measuring process performance and monitoring the efficacy of controls, and

- Continuously improving through the analysis of performance and the adoption of necessary process changes to achieve results.

Combining systems thinking with the co-creation of person centred care offers a powerful framework for redesigning healthcare. Healthcare can learn from other complex sectors where such systems based approaches have resulted in significant improvement: capitalising on the potential of person centred care and systems thinking will provide a significant step forward in addressing the health challenges of today and tomorrow.

Competing interests: We have read and understood BMJ policy on declaration of interests and declare DNV GL supports over 2200 healthcare providers around the world to apply systems thinking to address their risks and ensure the care they provide is quality focused and person centred. It does this through healthcare accreditation, managing infection risk certification, and other management system certification and training.

Provenance and peer review: Commissioned; not externally peer reviewed.

Donaldson L. In: Rasmussen E, Jørgensen K, Leyshon S, eds. Person-centred care-co-creating a healthcare sector for the future. Monday Morning Sustainia, 2014 
2 Boyle D, Harris M. The challenge of co-production: how equal partnerships between professionals and the public are crucial to improving public services. National Endowment for Science, Technology and the Arts, 2009.

3 Hibbard JH, Greene J, Overton V. Patients with lower activation associated with higher costs; delivery systems should know their patients' "scores." Health Aff 2013;32:216-22.

4 DiMatteo MR Giordani PJ, Lepper HS, Croghan TW. Patient adherence and medical treatment: a meta-analysis Med Care 2002;40:794-811.

5 Stewart M, Belle Brown J, Wetson WW, McWhinney IR, McWilliam C, Freeman TR. Patient-centered medicine: transforming the clinical method. Sage, 2003.
6 Foot C, Gilburt H, Dunn P, Jabbal J, Seale B, Goodrich J, et al. People in control of their own health and care. King's Fund, 2014.

De Savigny D, Adam T, eds. Systems thinking for health systems strengthening. WHO, 2009.

Cite this as: BMJ 2015;350:h985

(c) BMJ Publishing Group Ltd 2015 